\title{
Effets d'une supplémentation en méthionine ou en cystine de régimes carencés en acides aminés soufrés sur les performances de croissance du Lapin ( $\left.{ }^{1}\right)$
}

\author{
M. COLIN \\ avec la collaboration technique de G. SARDI \\ Labovatoive de Recherches sur l'Élevage du Lapin, \\ Centre national de Recherches zoolechniques, I.N.R.A., \\ $7835^{\circ}$ Jouy-en-Josas (France)
}

\begin{abstract}
Résumé
Au cours de deux expériences successives, on a recherché chez le Lapin en croissance, le pourcentage maximum d'acides aminés soufrés pouvant être apporté sous forme de cystine. I, a première expérience, réalisée avec 56 lapins californiens des deux sexes, a consisté à supplémenter un régime semi-purifié (contenant $0,42 \mathrm{p}$. cent d'acides aminés soufrés) à base de tourteau de soja, par 0,15 ou $0,3^{\circ} \mathrm{p}$. cent d'acides aminés soufrés libres (DL, méthionine ou $\mathrm{L}$ cystine ou ces 2 acides aminés en proportions égales). Les performances de croissance, de consommation et d'efficacité alimentaire des animaux ont été étudiées pendant 3 semaines.

Dans la seconde expérience, 92 lapins, de mêmes caractéristiques que ceux de la première expérience, ont été répartis entre 4 régimes alimentaires. L'aliment de base, composé essentiellement de tourteau de soja, de tourteau de tournesol, de luzerne déshydratée et d'amidon de maïs, apportait $0,44 \mathrm{p}$. cent d'acides aminés soufrés. Il était supplémenté par $0,20 \mathrm{p}$. cent de DL méthionine ou par $o, 20 \mathrm{p}$. cent de $\mathrm{L}$. cystine ou par o, Io $\mathrm{p}$. cent de DI, méthionine $+o, \mathrm{I} o$ p. cent de L cystine.

Dans tous les cas, l'apport d'acides aminés soufrés a fortement augmenté les performances des lapins et les supplémentations en DL, méthionine et en $L$ cystine ont eu des effets identiques.

Il semble donc que, chez le Lapin, la cystine puisse représenter 35 à 65 p. cent des acides aninés soụfrés du régime sans affecter les performances des animaux. Dans ces conditions, la cystine apportée par la plupart des aliments utilisés en pratique, est utilisée aussi efficacement que la méthionine et il convient donc de considérer un besoin global en acides aminés soufrés.
\end{abstract}

\section{Introduction}

L'étude des besoins en acides aminés soufrés du Lapin a fait l'objet de nombreuses expérimentations (CHEEKE, I971; AdAMson et Fisher, I973; Colin, Arkhurst et Ifebas, I973; Colin, I975; Davidson et Spreadbury, I975).' Mais

(I) Requests for reprints: Laboratoire de Recherches sur 1'Élevage du Lapin, I.N.R.A. B.P. 12, 31320 Castanet-Tolosan. 
dans tous ces travaux, les auteurs ont considéré que la méthionine et la cystine présentaient la même valeur nutritionnelle et ont donc formulé un besoin unique en acides aminés soufrés.

Or, on sait que chez le Porc (BAkER et al., I969; HENRy, RERAT et BourDon, I97I), le Rat (STOCKI,AND et al., r973), le Cobaye (REID, Ig66) comme chez le Poulet (Graber, Scort et BAKer, I97I), la cystine ne peut couvrir que 50 à 60 p. cent du besoin global en acides aminés soufrés.

Il convenait donc de vérifier que la cystine peut effectivement chez le Lapin remplacer une partie de la méthionine, et de déterminer le pourcentage maximum d'acides aminés soufrés pouvant être apportés sous forme de cystine dans les aliments destinés à cette espèce.

\section{Matériel et méthodes}

\section{Présentation des expériences}

La première expérience, réalisée sur 56 lapins, a consisté à comparer les effets de l'addition de DL méthionine ou de $\mathrm{L}$ cystine à un régime semi-purifié particulièrement carencé en acides aminés soufrés.

La seconde expérience, impliquant 92 lapins, a consisté à vérifier, dans le cas d'un aliment proche de ceux utilisés en pratique, les résultats obtenus dans la première expérience.

\section{Animaux}

L,es lapins utilisés, de race californienne et des deux sexes, étaient âgés de 35 jours en début d'expérimentation. Ils étaient logés en cages individuelles, entièrement métalliques et munies d'abreuvoir à surface d'eau libre.

\section{Déroulement des expériences}

La première expérience a duré 2 I jours, la seconde 35. La croissance des animaux a été déterminée par pesée hebdomadaire et leur consommation par pesée des quantités d'aliment distribuées et refusées, deux fois par semaine.

\section{Traitements et régimes expérimentaux}

Les aliments utilisés dans la $\mathbf{I}^{\text {re }}$ expérience sont de type semi purifié à base de tourteau de soja (tabl. I). Le régime de base, à I6 p. cent de matières azotées et 9,6 p. cent de cellulose brute, est fortement déficient en acides aminés soufrés $(0,42 \mathrm{p}$. cent seulement); sa concentration en énergie digestible est de $2850 \mathrm{kcal} / \mathrm{kg}$ (calculée). Quatorze lapins ont été affectés à ce traitement. Les 42 autres ont été répartis entre 6 régimes supplémentés en acides aminés soufrés correspondant à un schéma factoriel comprenant 2 taux d'acides aminés soufrés supplémentaires ( $0, I_{5}$ et $0,30 \mathrm{p}$. cent du régime) et 3 pourcentages de cystine parmi ces acides aminés supplémentaires (o, 50, roo p. cent) (7 lapins par aliment). 
TABLEAU I

Composition des régimes expérimentaux dans la premiève expérience Composition of the experimental diets used in the rst experiment

Acides aminés soufrés p. cent du régime (Sulphur amino acids p. cent of diet).

\begin{tabular}{|c|c|c|c|c|c|c|}
\hline $0,4^{2}$ & \multicolumn{3}{|c|}{0,57} & \multicolumn{3}{|c|}{0,72} \\
\hline 53 & 65 & 52 & 39 & 72 & $5 \mathrm{I}$ & $3^{\circ}$ \\
\hline 47 & 35 & $4^{8}$ & $6 \mathrm{I}$ & 28 & 49 & 70 \\
\hline 28 & 28 & 28 & 28 & 28 & 28 & 28 \\
\hline 25 & 25 & 25 & 25 & 25 & 25 & 25 \\
\hline 37,7 & 37,7 & 37,7 & 37,7 & 37,7 & 37,7 & 37,7 \\
\hline 3 & 3 & 3 & 3 & 3 & 3 & 3 \\
\hline- & & 0,075 & 0,15 & - & 0,15 & $0,3^{\circ}$ \\
\hline- & 0,15 & 0,075 & - & $0,3^{\circ}$ & o, I 5 & - \\
\hline 2,3 & $2, \mathrm{I}_{5}$ & 2,15 & 2,15 & 2,0 & 2,0 & 2,0 \\
\hline 4 & 4 & 4 & 4 & 4 & 4 & 4 \\
\hline
\end{tabular}

Cystine (p. cent des acides aminés soufrés) (Cystine (p. cent of S.A.A.)) . . . . . Méthionine (p. cent des acides aminés soufrés) (Methionine (p. cent of S.A.A.))

Tourteau de soja (1) (Soyabean meal (1)). 28

Paille d'orge (Barley straw) . . . . . . 25

Amidon de maïs (Maize starch). . . . . 37,7

Huile de maîs (Maize oil) . . . . . . . 3

DL, méthionine (DL methionine)..... -

I cystine (L cystine). . . . . . . . . . Glutamate de sodium (Sodium glutamate) CMV (') (Minerals and vitamins (2)) . .

(1) 49,4 p. cent de matières azotées. (49,4 p. cent of crude protein).

(j) Colin, Arkhurst et I,ebas (r973).

TABLEAU 2

Composition des régimes expérimentaux de la $z^{\mathrm{e}}$ expérience

Composition of the experimental diets used in the ad experiment

Acides aminés soufrés $\mathrm{p}$. cent du régime (Sulphur amino acids $p$. cent of diet $)$.......

Cystine (p. cent des acides aminés soufrés) (Cystine $($ p. cent of S.A.A.)) . . . . . . . . . . . . . .

Méthionine (p. cent des acides aminés soufrés) (Methionine $(p$. cent of $S . A . A))$. . . .

Tourteau de soja $\left(^{1}\right)$ (Soyabean meal) . . . . . . . .

Tourteau de tournesol $\left({ }^{2}\right)$ (Sunflower meal). . . . . . Luzerne déshydratée ${ }^{3}$ ) (Dehydrated lucerne), . . . . Amidon de maïs (Maize starch). . . . . . . . . . . . . . . .

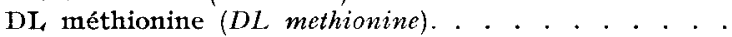

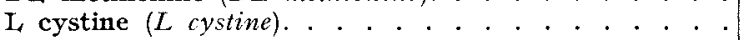
Glutamate de sodium (Sodium glutamate). . . . . .

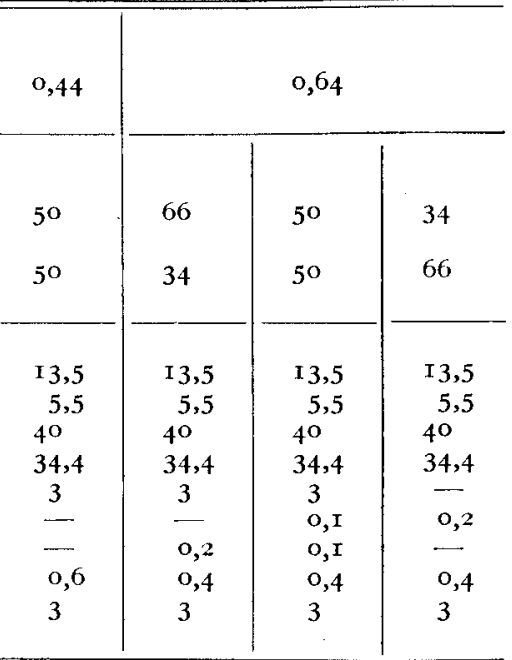
CMV ( $\left.{ }^{4}\right)$ (Minerals and vitamins) . . . . . . . . . .

(1) 47,8 p. cent de matières azotées (of crude protein).

(2) $34 \mathrm{p}$. cent de matières azotées (of crude protein).

(3) 16,9 p. cent de matières azotées (of crude protein).

(4) LeBas et ColiN (I977). 
Les aliments utilisés dans la seconde expérience (tabl. 2) étaient essentiellement à base de tourteau de soja, de tourteau de tournesol, de luzerne déshydratée et d'amidon de mais. Le régime de base contenant ${ }_{5} 5,4 \mathrm{p}$. cent de matières azotées et II,8 p. cent de cellulose brute apportait $0,44 \mathrm{p}$. cent d'acides aminés soufrés et $2650 \mathrm{Kcal} \mathrm{E.D.} / \mathrm{kg}$. Il était supplémenté respectivement par $0,2 \mathrm{p}$. cent de L cystine ou o, I p. cent de $\mathrm{L}$ cystine + o,I p. cent de DL méthionine ou encore 0,2 p. cent de DL méthionine. Vingt-trois lapins ont été affectés à chacun de ces traitements.

Dans les deux expériences, les valeurs obtenues pour chaque traitement ont été comparées entre elles par analyse de variance, puis par un test de comparaison multiple de moyennes de Newman et Keuls,

\section{Résultats}

\section{Première expérience}

La supplémentation du régime de base par la métbionine et la cystine a très fortement augmenté la croissance des lapins $\left(+4^{0} \mathrm{a}+55 \mathrm{p}\right.$. cent), de même que leur consommation $(+20$ à +30 p. cent) (tabl. 3). L'indice de consommation a également été amélioré de io à $20 \mathrm{p}$. cent. Globalement, on observe très peu de différences entre les résultats correspondant aux 6 modalités de supplémentation. Les croissances obtenues étaient cependant très légèrement plus élevées lorsque le régime contenait $0,7^{2} \mathrm{p}$. cent d'acides aminés soufrés que lorsqu'il en apportait $0,57 \mathrm{p}$. cent (Différence significative au seuil $\mathrm{P}=0, \mathrm{r} 0$ ).

De même, les lapins ayant reçu de l'aliment supplémenté de DL méthionine seule ont eu un indice de consommation plus bas que ceux affectés aux rations supplémentaires totalement ou partiellement par de la cystine (I)ifférence significative au seuil $\mathrm{P}=\% 0,05)$.

\section{Seconde expérience}

La supplémentation par les acides aminés soufrés libres a également augmenté les performances de croissance $(+$ Io à + I 5 p. cent) et de consommation $(+5$ à + Io p. cent) (tabl. 4). Au contraire des observations réalisées lors de la première expérience, l'indice de consommation n'a pratiquement pas été modifié. On n'observe aucune différence entre les 3 modalités de supplémientation (DL méthionine seule ou $\mathrm{L}$ cystine seule ou $50 \mathrm{p}$. cent $\mathrm{DL}$ méthionine $+50 \mathrm{p}$. cent $\mathrm{L}$ cystine).

\section{Discussion}

Comme dans les travaux antérieurs réalisés avec des régimes du même type (Colin, Arkhurst et LEBas, I973; Colin, I975), 1'incorporation de DL méthionine à un régime apportant moins de $0,45 \mathrm{p}$. cent d'acides aminés soufrés améliore très fortement les performances de croissance des lapins. Le remplacement total ou partiel de cette DL méthionine additionnelle par de la $\mathrm{L}$ cystine n'entraîne 


\begin{tabular}{|c|c|c|c|c|c|c|c|}
\hline 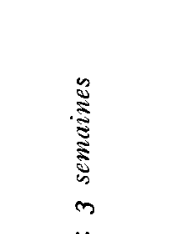 & 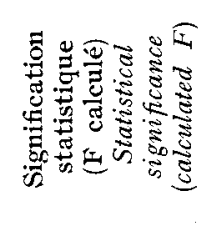 & & $\begin{array}{l}\frac{\bar{a}}{2} \\
\frac{\pi}{2} \\
\bar{v} \\
v\end{array}$ & 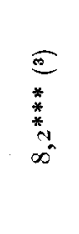 & 草 & 莣 & 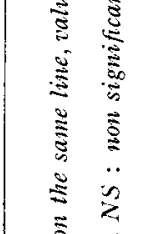 \\
\hline 竎 & \multirow{3}{*}{0} & $\dot{m} i$ & 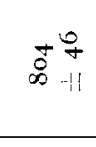 & 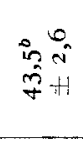 & 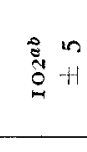 & 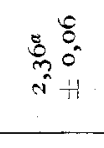 & $\begin{array}{l}\overrightarrow{0} \\
0 \\
0\end{array}$ \\
\hline 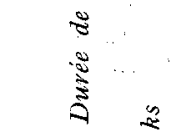 & & in $q$ & $\begin{array}{l}\stackrel{0}{7} \\
80.11\end{array}$ & $\begin{array}{l}8=0 \\
0=5 \\
6=-11\end{array}$ & $i_{i=1}^{m}$ & 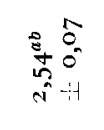 & $\begin{array}{ll}\bar{\Xi} \\
\bar{\Xi} \\
\bar{z}\end{array}$ \\
\hline 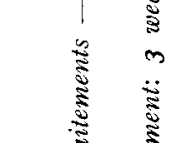 & & $\therefore \quad \stackrel{\infty}{i}$ & $\stackrel{8}{x}^{7}$ & 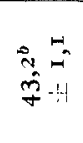 & $\stackrel{8}{\square}$ & 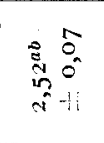 & 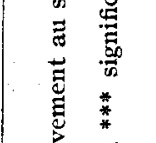 \\
\hline 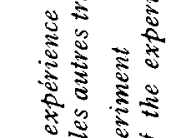 & \multirow{3}{*}{ o } & $\dot{m} \bar{b}$ & 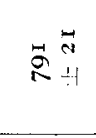 & $\begin{array}{l}0_{0} \hat{A} \\
\hat{y}+1\end{array}$ & 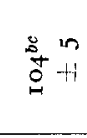 & 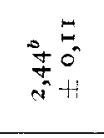 & 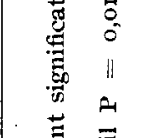 \\
\hline 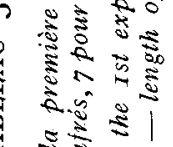 & & in $\stackrel{\infty}{+}$ & $\stackrel{2}{2}$ & $\hat{\vec{y}} \hat{\bar{y}}$ & 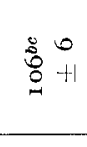 & 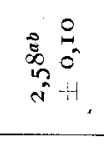 & 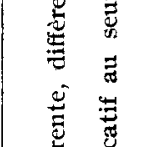 \\
\hline 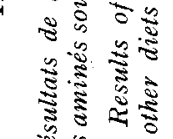 & & $8 \%$ & $\hat{\infty} \begin{array}{c}\hat{m} \\
\infty\end{array}$ & 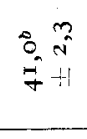 & $\begin{array}{c}0 \\
0 \\
0 \\
0 \\
0\end{array}$ & 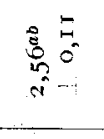 & 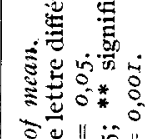 \\
\hline 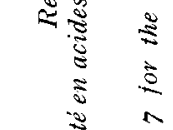 & $\begin{array}{l}7 \\
0 \\
0\end{array}$ & $n$ f & 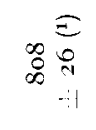 & 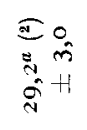 & 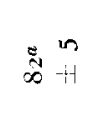 & 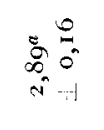 & \\
\hline 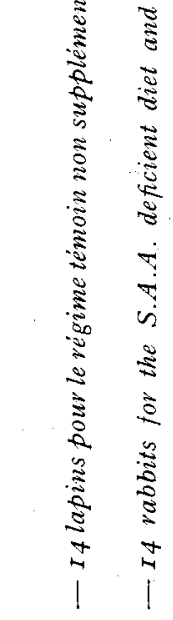 & 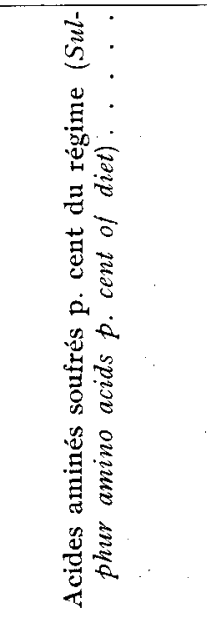 & 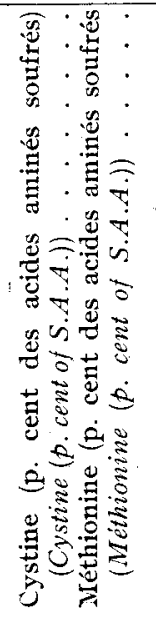 & 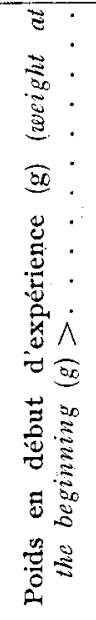 & 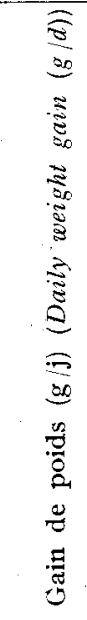 & 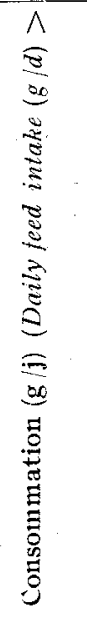 & 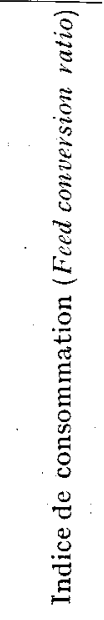 & 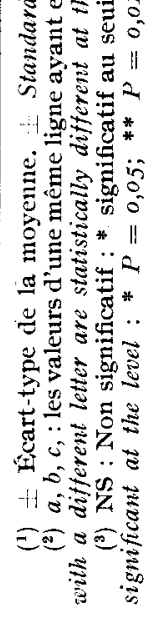 \\
\hline
\end{tabular}


Résultats de la seconde expérience :

- 23 lapins par régime,

- duvée de l'expérience : 5 semaines.

Results of the $2 d$ experiment:

- 23 rabbits per treatment,

- length of the experiment: 5 weeks.

\begin{tabular}{|c|c|c|c|c|c|}
\hline 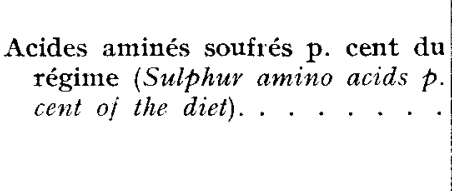 & 0,44 & & 0,64 & & $\begin{array}{l}\text { Signification } \\
\text { statistique } \\
(\mathrm{F} \text { calculé) } \\
\text { Statistical } \\
\text { significance } \\
\text { (calculated } F) \text { ) }\end{array}$ \\
\hline 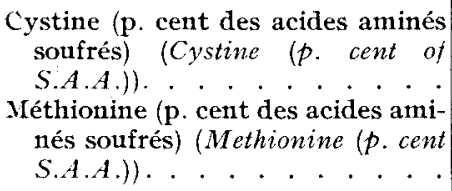 & $5^{\circ}$ & 66 & $5^{\circ}$ & 34 & \\
\hline $\begin{array}{l}\text { Poids en début d'expérience }(\mathrm{g}) \\
\text { (Weight at the beginning }(g)) \\
\text { Gain de poids }(\mathrm{g} / \mathrm{j}) \text { (Daily weight } \\
\text { gain }(g / d)) \cdot \cdot \cdot \cdot \cdot \cdot \cdot \cdot \\
\text { Consommation }(\mathrm{g} / \mathrm{j})(\text { Daily feed in- } \\
\text { take }(g / d)) \cdot \cdot \cdot \cdot \cdot \cdot \cdot \cdot \cdot \\
\text { Indice de consommation (Feed } \\
\text { conversion ratio)........ }\end{array}$ & $\begin{array}{c}87 \mathrm{r} \\
\pm \mathrm{I} 5{ }^{(1)} \\
33,0^{a}{ }^{(2)} \\
\pm 0,7 \\
95^{\mathrm{a}} \\
\pm 2 \\
2,90 \\
\pm 0,05\end{array}$ & $\begin{array}{l}87^{0} \\
\pm 14 \\
\\
37,2^{b} \\
\pm 0,8 \\
105^{b} \\
\pm 3 \\
2,81 \\
\therefore 0,07\end{array}$ & 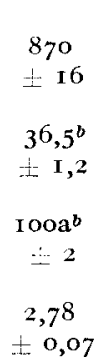 & 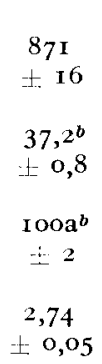 & $\begin{array}{l}<\mathrm{INS}\left({ }^{3}\right) \\
4,96^{* *}\left({ }^{3}\right) \\
2,75^{*}\left({ }^{3}\right) \\
<\mathrm{I} \text { NS }\end{array}$ \\
\hline
\end{tabular}

(1) (2) (3): Voir tablean 3. Sce table 3 .

aucune modification du gain de poids. Toutefois, les résultats de la première expérience pourraient laisser penser que la substitution de la DL méthionine par la $\mathrm{L}$, cystine entraîne une légère dégradation de l'indice de consommation (5 à 7 p. cent). Cependant, cet effet n'a pas été retrouvé dans la seconde expérience.

Des essais ultérieurs devront préciser si les écarts observés ici au cours de la première expérience reflètent un phénomène existant réellement (dont l'impor.tance quantitative resterait de toute façon très faible) ou s'ils sont de nature aléatoire.

Globalement, il semble donc que chez le lapin la cystine peut représenter 35 à 65 p. cent des acides aminés soufrés du régime sans affecter les performances de croissance de l'animal. En particulier, on n'observe aucun effet dépressif lorsque plus de 60 p. cent des acides aminés soufrés de 1'aliment sont apportés sous forme de cystine. Le lapin paraît donc moins sensible à l'équilibre entre méthionine 
et cystine que le porc (BAKER et al., I969; HENRY, RERAT et BOURdon, I97I) ou le poulet (Graber, ScotT et BAKER, I97I).

En pratique, compte tenu des teneurs en méthionine et en cystine des matières premières utilisées pour 1'alimentation du lapin (PION et FAUCONNEAU, I966; HARVEY, 1970), on peut considérer que la cystine est utilisée aussi efficacement que la méthionine dans la quasi-totalité des rations distribuées aux lapins. En ce sens, il convient bien de considérer, pour une croissance pondérale maximale, un besoin global en acides aminés soufrés du lapin en croissance.

Les résultats rapportés ici, en accord avec la plupart des travaux antérieurs (Colin, Arkhurst et Lebas, I973; Colin, r975; Davidson et Spreadbury, I975) montrent que ce besoin est compris entre 0,55 et $0,65 \mathrm{p}$. cent du régime, ces fluctuations étant en grande partie en relation avec la concentration énergétique des aliments (Cor.is, I975, I977).

Accepte pour publication en novembre 1977.

\section{Summary}

\section{Effects of methionine or cystine supplementation of sulphur amino acid deficient diets on the growth performance of rabbits}

During two successive experiments, attempts were made to determine the maximum sulphur amino acid supplementation of growing rabbit diets in form of cystine. The first experiment made with 56 Californian rabbits of both sexes involved a supplementation of a semipurified diet (containing $0.4^{2}$ p. cent sulphur amino acids) based on soyabean meal with 0 .I 5 or $0.30 \mathrm{p}$. cent free sulphur amino acids (DL methionine or L cystine or these two amino acids in equal proportions). Growth, feed intake and feed efficiency were studied in the animals for a period of 3 weeks.

In the second experiment, 92 rabbits with the same characteristics as those of the first experiment, were allocated to 4 treatments. The basal diet, mainly composed of soyabean meal, sunflower meal, dried lucerne and starch, supplied $0.44 \mathrm{p}$. cent sulphur amino acids. It was supplemented with $0.20 \mathrm{p}$. cent DL methionine or $0.20 \mathrm{p}$. cent $\mathrm{L}$ cystine or o.Io p. cent DI. methionine +0.10 p. cent cystine.

In all cases, the supply of sulphur amino acids strongly increased the performances of the rabbits; DI, methionine and $\mathrm{L}$ cystine supplementation had the same effects.

It therefore seems that in the rabbit, cystine may represent 35 to $65 \mathrm{p}$. cent of the sulphur amino acids without affecting the performances of the animals. Under these conditions, cystine included in most of the feeds used in practice is as efficiently used as methionine. Accordingly, for obtaining maximum growth performances in the rabbit, it is advisable to consider the overall requirement for sulphur amino acids.

\section{Références bibliographiques}

ADAMSON I., lisher H., 1973. The aminoacid requirement of the growing rabbit: an estimate of quantitative needs. J. Nutr., 103, I 306-1319.

Bakle D. H., Clausing W. W., Harmon B. G., Jensin A. H., Becker D. E., ig6g. Replacement value of cystine for methionine for the young pig. J. anim. Sci., 29, 58I-584.

CHEEkE P. R., I97I. Arginine, lysine and methionine needs of the growing rabbit. Nutr. Rept. Int., 3, I $23-\mathrm{I} 28$.

Colin M., 1975. Fffets sur la croissance du Lapin de la supplémentation en L-lysine et DL-méthionine de régimes régétaux simplifiés. Ann. Zootech., 24, 465-474 
Colin M., ALLAIN D., I978. Fitude du besoin en lysine du Lapin en croissance en relation avec la concentration énergétique de l'aliment. Ann. Zootech., 27, i 7-31.

Colin M., Arkhurst G., LEbas F., 1973. Effets de l'addition de méthionine au régime alimentaire sur les performances de croissance du Lapin. Ann. Zootech., 22, 485-49I.

Davidsox J., Spreadbury D., 1975. Nutrition of the New Zealand White rabbit. Proc. Nutv. Soc., 34, 75-83.

Graber G., SCOTT H. M., BakER D.H., I97I. Sulphur amino acid nutrition of the growing chick: effect of age on the capacity of cystine to spare dietary methionine. Poultry Sci., 50, I $450-I_{455}$.

HARVEY D., 1970. Tables of the amino, acids in foods and feedingstuffs, 105 pages. Commonwealth agricultural bureaux, Farnham Royal Bucks (England).

Henry Y., Rerat A., Bourdon D., r971. Efféts comparés d'une supplémentation en méthionine et en cystine sur les performances de croissance et la composition corporelle du Porc. $I^{\mathrm{C}}$ Congrès International de Zootechnie, Versailles.

LEBAS F., Colin M., I977. Utilisation du tourteau de colza dans l'alimentation du Lapin en croissance, influence du dépelliculage. Ann. Zootech., 26, 93-97.

Pion R., Fauconneau G., I966. Les acides aminés des protéines alimentaires. Méthode de dosage et résultats obtenus. Cahier A.E.C. $n^{\circ} 6$ "Amino acides, peptides, protéines" ", I55-I 75 .

REID M. E., I966. Methionine and cystine requirement of the young guinea-pig. $J$. Nutr., 88, 397-402.

Stockiand W. I., MEade R. J., Wass D. F., Sowress J. E., 1973. Influence of the levels of methionine and cystine on the total sulphur amino acid requirement of the growing rat. $J$. anim. Sci., 36, 526-530. 